\title{
Erratum to: Metformin increases phagocytosis and acidifies lysosomal/endosomal compartments in AMPK-dependent manner in rat primary microglia
}

\author{
Krzysztof Labuzek $^{1}$ - Sebastian Liber ${ }^{1}$ - Bożena Gabryel ${ }^{2}$ - Jakub Adamczyk ${ }^{3}$. \\ Boguslaw Okopień ${ }^{1}$
}

Published online: 4 February 2017

(C) Springer-Verlag Berlin Heidelberg 2017

\section{Erratum to: Naunyn-Schmiedeberg's Arch Pharmacol}

DOI 10.1007/s00210-009-0477-x

The authors regret that the Figure 7 containing representative western blots is not of sufficient quality and should be replaced. In the final steps of figure preparation the graphics technician made an incorrect insertion of blots into figure 7 , which probably resulted from relatively low resolution of images.

In order to meet high standard of Naunyn-Schmiedebergs Archives of Pharmacology and to improve clarity of data presentation we wish to perform a corrigendum of figure 7, which would only affect graphical representation of WB bands and not scientific merit.

The authors would like to apologise for any inconvenience caused.

The online version of the original article can be found at doi:10.1007/ s00210-009-0477-x.

Krzysztof Łabuzek

labuzek@labuzek.com

1 Department of Clinical Pharmacology, Medical University of Silesia, Medyków 18, PL 40-752 Katowice, Poland

2 Department of Pharmacology, Medical University of Silesia, Medyków 18, PL 40-752 Katowice, Poland

3 Department of Biophysics, School of Pharmacy, Medical University of Silesia, Sosnowiec, Poland 


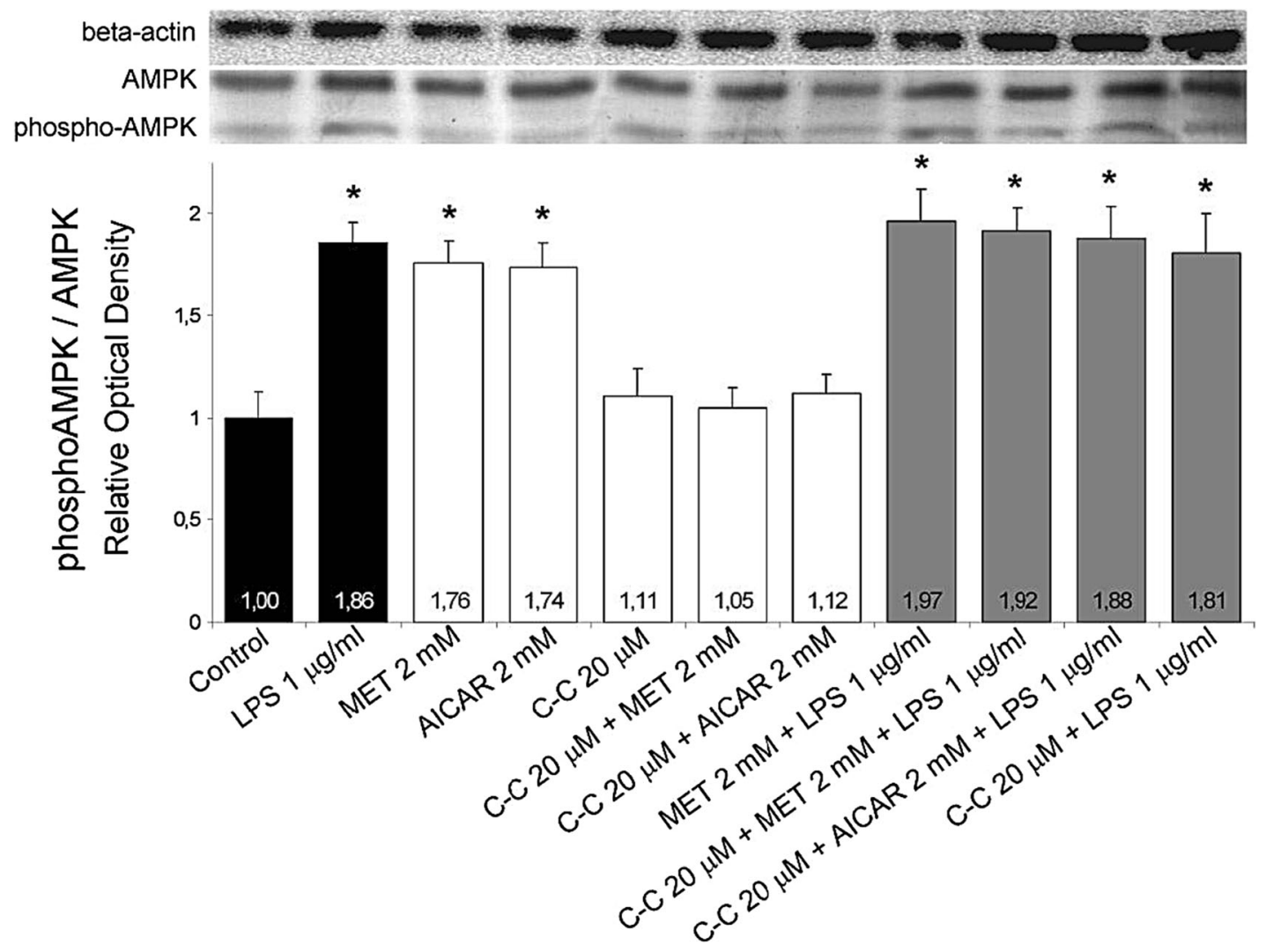

